\title{
CHRONOBIOLOGY OF DEPRESSION
}

\section{CHRONOBIOLOGIE DER DEPRESSION}

\author{
Lucie Bartova $^{1}$ \& Siegfried Kasper ${ }^{1,2}$ \\ ${ }^{1}$ Klinische Abteilung für Allgemeine Psychiatrie, Universitätsklinik für Psychiatrie und Psychotherapie, \\ Medizinische Universität Wien, Wien, Österreich \\ ${ }^{2}$ Zentrum für Hirnforschung, Medizinische Universität Wien, Wien, Österreich
}

\section{SUMMARY}

Seasonal fluctuations in mood, drive, energy, sleepingand eating behavior, weight, as well as further important mental and physical functions, and the utilization of light as an effective treatment option were already described by Hippocrates of Kos and Araeteus, the Cappadocian. The concept of the so-called seasonal affective disorder (SAD) as a disruption of the circadian rhythm precipitated by a deficiency of environmental light during darker seasons was first described in the 1980s. Furthermore, chronobiological and hormonal dysregulation in SAD patients was repeatedly shown to be accompanied by alterations on a neuroreceptor and neurotransmitter level and to normalize after remission. Hence, SAD represents one of the most important models of a chronobiological disorder with over 1000 international publications on its aetiology and treatment options, whereby their underpinnings could be elucidated on a clinical as well as molecular level.

The present article summarizes the current understanding of etiological mechanisms of SAD and provides an overview of diagnostic and therapeutic strategies, which are based on available international evidence including clinical trials, systematic reviews, and meta-analyses. According to current recommendations of international guidelines, promising treatment options as bright light therapy, psychopharmacotherapy, therapeutic sleep deprivation, and their underlying mechanisms of action are presented.

Key words: seasonal affective disorder - winter depression - bright light therapy - therapeutic sleep deprivation antidepressants

\section{ZUSAMMENFASSUNG}

Jahreszeitabhängige Schwankungen von Stimmung, Antrieb, Energieniveau, Schlafdauer, Essverhalten, Körpergewicht, sowie von weiteren wichtigen psychischen und körperlichen Funktionen, und deren Behandlungsmöglichkeit mit Licht wurden schon von Hippocrates von Kos und Araeteus, dem Kappadokier, beschrieben. In den 1980er Jahren wurde das Konzept der sogenannten saisonal abhängigen affektiven Störung (SAD) als eine Störung des zirkadianen Rhythmus vorgestellt, welche durch Mangel an Umgebungslicht in den dunkleren Jahreszeiten hervorgerufen wird. Außerdem konnte gezeigt werden, dass chronobiologische und hormonelle Abweichungen bei PatientInnen mit einer SAD mit Veränderungen auf der Neurorezeptor- und Neurotransmitterebene einhergehen, welche sich nach Erreichen einer Remission wieder normalisieren. Die SAD stellt somit eines der wichtigsten Modelle einer chronobiologischen Störung dar mit nun weit über 1000 internationalen Veröffentlichungen zu deren Ätiologie und Therapiemöglichkeiten, deren zugrundeliegenden Mechanismen sowohl klinisch als auch auf molekularer Ebene beschrieben werden können.

Dieser Artikel bietet eine Zusammenfassung unseres gegenwärtigen Verständnisses über die Ätiologie der SAD sowie eine Übersicht von aktuellen diagnostischen und therapeutischen Strategien, welche anhand von Ergebnissen der derzeit verfügbaren klinischen Studien, systematischen Reviews, Meta-Analysen, und im Einklang mit aktuellen international anerkannten Richtlinien erstellt wurde. Hierbei werden vielversprechende Behandlungsoptionen wie die Lichttherapie, Psychopharmakotherapie, sowie die Wachtherapie bzw. der therapeutische Schlafentzug und deren zugrundeliegenden Wirkmechanismen erläutert.

Schlüsselwörter: Saisonal abhängige affektive Störung Herbst-Winter-Depression - Lichttherapie - Wachtherapie (Schlafentzugstherapie) - Antidepressiva

\section{EINLEITUNG}

Nachdem das Leben bekanntlich durch endogene biologische Rhythmen determiniert ist, variieren viele wichtige Funktionen des menschlichen Körpers physiologisch in circatidalen (12h), circadianen (24h), circalunaren (28d), circaannualen (365d) und weiteren Perioden. Zahlreiche Untersuchungen, welche sowohl an Tieren als auch an Menschen durchgeführt wurden, konnten zeigen, dass sich z. B. die Stimmung, Aufmerksamkeit, Reaktionszeit und Koordination, die kardiovaskuläre Leistung und Muskelkraft, die Darmperistaltik, die Körpertemperatur, sowie die Sekretion des Stresshormons Cortisol, des Sexualhormons Testosteron, des Wachst- umshormons Somatotropin, und des für die Steuerung des Tag-Nacht-Rhythmus verantwortlichen Hormons Melatonin im Tagesverlauf unterscheiden (Linkowski et al. 1987, Souetre et al. 1989, Peeters et al. 2006, Murray 2007, Lehofer et al. 2010). Hierbei ist z. B. bekannt, dass das allgemeine Wohlbefinden abends steigt, die Körpertemperatur am Abend am höchsten ist, oder dass Schmerzen in der Früh am stärksten wahrgenommen werden. Während Melatonin und Somatotropin in der Nacht ausgeschüttet werden, erfahren Cortisol- und Testosteronsekretion ihre Höchstleistung in der Früh. Es ist von besonderer Bedeutung, dass die o. g. Hormonsekretion und die zusammenhängenden Funktionen, welche für einen einwandfreien Lebensablauf unent- 
behrlich sind, durch den Nuclueus suprachiasmaticus (SCN) in Abhängigkeit von Licht mit dem 24-StundenTagrhythmus synchronisiert werden (Liebmann et al. 1997; Mendoza 2019). Der SCN befindet sich in der anterioren hypothalamischen Region und wird häufig als ,innere Uhr“" bzw. „zentraler Schrittmacher des zirkadianen Systems“ bezeichnet (Lehofer et al. 2010).

Neben der wichtigen tagesrhythmischen Wirkung beeinflusst Licht unseren Organismus signifikant auch über längere Zeitperioden. Die menschliche Physiologie zeigt hierbei klare jahreszeitliche Adaptationsprozesse und Schwankungen in vielen körperlichen und psychischen Funktionen. Bei einem Großteil der Menschen, die in gemäßigten Klimazonen leben und somit einen regelmäßigen Wechsel von vier Jahreszeiten mit Variabilität in der Stundenanzahl täglicher Sonneneinstrahlung erleben, kommt es hierbei in unterschiedlicher Ausprägung $\mathrm{zu}$ jahreszeitlichen Veränderungen von Schlafdauer, Essverhalten, Körpergewicht, Energieniveau, sowie Antrieb und Stimmungslage (Kasper et al. 1989). Es scheint sogar eine allgemeine Erfahrung zu sein, dass sich viele Menschen an hellen und sonnigen Tagen energiegeladener und besser gelaunt fühlen als an dunklen Tagen (Lehofer et al. 2010). Dies ist von essentieller Relevanz, da eine ausgeglichene Stimmung und ein zufriedenstellendes Energieniveau sowie Schlafund Essverhalten oft unser Wohlbefinden bestimmen und bei zahlreichen Erkrankungen in unterschiedlicher Art und Weise verändert sind.

\section{CHRONOBIOLOGIE DER DEPRESSION UND SUIZIDALITÄT}

Bei affektiven Erkrankungen zählen pathologische Veränderungen der Stimmung und der Befindlichkeit, der Impulsivität, des Antriebs, des Appetits und des Körpergewichts, sowie des Schlafverhaltens, welche weiters mit Aggression und/oder Suizidalität häufig einhergehen können, zu den häufigsten Symptomen (Kasper et al. 1989; Winkler et al. 2006b), welche u. a. durch den Neurotransmitter Serotonin (5-Hydroxytryptamin, 5-HT) moduliert werden (Willeit et al. 2000; Neumeister et al. 2001; Wehr et al. 2001). So wie Melatonin gehört auch Serotonin zur Gruppe der sogenannten Indolamine, welche seit der frühen Evolution Lichtsignale und Informationen über die Photoperiode in Zellen und Organismen übertragen, und daher eine wichtige Rolle als Signalgeber für zeitlich bedingte physiologische sowie pathologische Veränderungen in den o. g. Funktionsbereichen spielen (Willeit et al. 2000; Neumeister et al. 2001; Wehr et al. 2001; Lehofer et al. 2010).

Im Rahmen der zirkadianen Rhythmik der depressiven Symptomatik ist das sogenannte Morgenpessimum charakteristisch. Außerdem zeigen depressive PatientInnen im Vergleich zu Nichterkrankten häufig weitere zirkadiane Rhythmusstörungen, wie z. B. die Abflachung der Körpertemperatur im Tagesverlauf, eine Phasenvorverschiebung der Sekretion von Cortisol, sowie ein fehlendes nächtliches Ansteigen der Plasmakonzentration von Melatonin (Linkowski et al. 1987; Wittmann et al. 2018). In diesem Kontext ist erwähnenswert, dass etwa 90\% aller depressiven PatientInnen unter Schlafstörungen leiden, welche im Schlaflabor mit verkürzter Latenz bis zur initialen REM-Phase, vermehrtem REM-Schlaf zu Beginn der Nacht, sowie einer insgesamt reduzierten Tiefschlafdauer korrelieren (Lehofer et al. 2010; Kudlow et al. 2013).

Im Rahmen der saisonalen Rhythmik der depressiven Symptomatik ist die jahreszeitliche Schwankung der Suizidhäufigkeit mit punctum maximum im Frühling von besonderer Bedeutung (Lambert et al. 2002; Lambert et al. 2003). Diese wurde in gemäßigten Breiten wiederholt verzeichnet, wo es $\mathrm{zu}$ einem regelmäßigen Jahreszeitenwechsel mit nachweislichen Veränderungen in der täglichen Stundenanzahl der Sonneneinstrahlung kommt, während in tropischen Regionen mit täglich-konstanter Stundenanzahl der Sonneneinstrahlung keine jahreszeitliche Häufung der Suizide bisher beobachtet wurde (Kasper et al. 1989; Hardin et al. 1991; Harris \& Dawson-Hughes 1993; Hegde \& Woodson 1996; Okawa et al. 1996; Rohan \& Sigmon 2000; Perry et al. 2001; Chotai et al. 2004; Friedman et al. 2006; Vyssoki et al. 2012, Vyssoki et al. 2014).

Anhand verfügbarer Studienergebnisse geht man derzeit davon aus, dass die saisonale Steigerung der Suizidhäufigkeit im Frühling mit einer parallelen $\mathrm{Zu}-$ nahme der Impulsivität einhergeht, welche mit zunehmender Stundenanzahl täglicher Sonneneinstrahlung steigt (Lambert et al. 2002, Lambert et al. 2003). Als ein möglicher zugrundeliegender neurobiologischer Mechanismus wurde hierbei die Abnahme der 5-HT-Transporter (5-HTT)-Bindung im Frühling beschrieben (Praschak-Rieder et al. 2008). Im Detail konnten hohe Impulsivitätswerte mit einer niedrigen 5-HTT-Bindung in bildgebenden Studien assoziiert werden, während Untersuchungen, welche post-mortem durchgeführt wurden, erniedrigte 5-HTT-Dichte im Gehirn von Suizidopfern nachweisen konnten (Bach \& Arango 2012). Von besonderer Bedeutung ist hierbei, dass es durch zunehmende Verordnungsdichte antidepressiv wirkender Substanzen, welche ihre antidepressive Wirksamkeit großteils durch die direkte Beeinflussung des 5-HT-Stoffwechsels (Antagonismus an 5-HTRezeptoren, Hemmung der 5-HT-Wiederaufnahme, Hemmung der Monoaminoxidase) entfalten, neben einer allgemeinen Reduktion der Suizidrate auch zu einer Abflachung des Suizidgipfels im Frühjahr kam (Lehofer et al. 2010; Sebestyen et al. 2010).

\section{SAISONAL ABHÄNGIGE AFFEKTIVE STÖRUNG}

Die saisonal abhängige affektive Störung (SAD) stellt einen Subtyp der rezidivierenden depressiven Störung (75\%) oder der bipolaren Störung (25\%) dar, die durch das regelmäßige (über mindestens 2 Jahre) 
und zeitlich alternierende Auftreten affektiver Episoden zu einer bestimmten Jahreszeit definiert wird (APA, 2013). Im Rahmen des SAD Spektrums ist die sogenannte Herbst-Winter-Depression mit Beginn im Herbst oder Winter und einer spontanen Remission oder seltener Hypomanie/Manie im darauffolgenden Frühjahr oder Sommer am häufigsten (Rosenthal et al. 1984). In seltenen Fällen zeigt sich auch ein umgekehrter Verlauf mit dem Auftreten depressiver Symptome im Frühling oder Sommer (Wehr et al. 1987).

Um eine SAD korrekt zu diagnostizieren sollten die saisonal abhängigen affektiven Episoden die Anzahl der nicht saisonalen Episoden eindeutig übersteigen. Die sogenannte saisonal abhängige Depression tritt üblicherweise Jahr für Jahr während der Herbst- und Wintermonate auf und zeichnet sich neben den für die depressive Störung typischen Symptomen wie z. B. depressive Stimmung, Antriebslosigkeit, Anhedonie und Konzentrationsstörungen auch durch atypische vegetative Symptome wie z. B. Appetitsteigerung, Kohlehydratheißhunger und anschließende Gewichtszunahme aus (Kasper et al. 1988a, 1989, Praschak-Rieder et al. 2005a). Die Mehrheit der SAD- PatientInnen erfüllen die Kriterien des sogenannten atypischen Subtyps einer depressiven Störung, welcher durch Symptome wie z. B. bleierne Schwere, Irritabilität, und Hypersomnie charakterisiert wird (Kasper et al. 1988a, Winkler et al. 2002b).

\section{EPIDEMIOLOGIE DER SAD}

Bezugnehmend auf die Allgemeinbevölkerung leiden derzeit 2-4\% der Menschen unter einer SAD, während $8-12 \%$ die sogenannte subsyndromale SAD aufweisen, welche durch dieselbe Symptomatik in einer weniger intensiven Ausprägung charakterisiert wird (Kasper 1994; Lehofer et al. 2010). Analog zu Studien zur Suizidalität wurde eine höhere Prävalenz der SAD in gemäßigten Breiten mit einem regelmäßigen Jahreszeitenwechsel berichtet (Kasper et al. 1989; Levitt \& Boyle 2002). Untersuchungen, welche in Mitteleuropa durchgeführt wurden, zeigten, dass sich etwa jeder fünfte Mensch in irgendeiner Form durch die dunkle Jahreszeit in seinem Wohlbefinden beeinträchtigt fühlt (Lehofer et al. 2010). In Bezug auf die depressive Störung im Allgemeinen ist erwähnenswert, dass etwa $11 \%$ diagnostizierter depressiver Phasen im Rahmen einer SAD auftreten (Levitt et al. 2000). Frauen sind hierbei drei- bis fünfmal häufiger betroffen als Männer, wobei das Verhältnis zwischen Frauen und Männern genau wie bei einer nicht-saisonalen Depression bei ca. 2:1 liegt (Winkler et al. 2002a).

\section{PATHOGENESE DER SAD}

Aktuell geht man davon aus, dass ein Lichtmangel in der dunklen Jahreszeit zusammen mit einer genetischen Veranlagung die Ursache der SAD darstellen (Kasper et al. 1988a, 1989). Genetisch fokussierte Studien konnten bereits wiederholt auf die Heritabilität der SAD und
Saisonalität im Allgemeinen mit einer individuellen Vulnerabilität hinweisen (Praschak-Rieder et al. 2002, Willeit et al. 2003, Praschak-Rieder et al. 2005b). Hierbei konnte gezeigt werden, dass genetische Faktoren eine bedeutende Rolle für die individuelle Empfindlichkeit gegenüber jahreszeitlichen Veränderungen spielen. Im Vergleich $\mathrm{zu}$ entsprechend vulnerablen Individuen scheinen Menschen, welche gegenüber Veränderungen von Umgebungsfaktoren wie z. B. Licht oder Temperatur resilient sind, weniger leicht an einer $\mathrm{SAD} z \mathrm{zu}$ erkranken (Kasper et al. 1988a, 1989).

Zur Pathogenese der SAD werden derzeit zwei wichtige und sich ergänzende Hypothesen diskutiert. Die photoneurochemische Hypothese basiert auf der Tatsache, dass Licht über die Netzhaut der Augen aufgenommen wird und weiter über glutamaterge Neurone an das Gehirn weitergeleitet wird. Hierbei ist von besonderer Bedeutung, dass die Sehnerven eine Abzweigung zum SCN besitzen, welcher wichtige endogene Rhythmen wie z. B. die Hormonproduktion und den Schlaf-Wach-Rhythmus mit dem äußeren Hell-DunkelZyklus synchronisiert. Im Rahmen dieser komplexen Prozesse spielt $u$. a. die serotonerge Neurotransmission eine bedeutende Rolle (Praschak-Rieder et al. 2008; Willeit et al. 2008; Praschak-Rieder \& Willeit 2012). Die Phasenverschiebungshypothese beruht auf der Tatsache der zahlreich beobachteten verschobenen zirkadianen Phase bei SAD PatientInnen, welche sich nach einer erfolgreichen Anwendung morgendlicher Lichttherapie, die zu einer Phasenvorverschiebung führt, wieder normalisiert (Terman et al. 2001, Winkler et al. 2005, Pail et al. 2011).

\section{THERAPIE DER SAD}

So wie bei jeder anderen medizinischen Erkrankung sollte die Behandlung der SAD entsprechend der individuellen Bedürfnisse der Betroffenen gestaltet werden. Hierbei stellen regelmäßige Psychoedukation und eine tragfähige Arzt-PatientInnen Beziehung die Voraussetzung für eine effektive, gut verträgliche, therapieadhärenzfördernde und somit erfolgreiche Behandlung dar. Neben der Lichttherapie, welche gegenwärtig die first-line Strategie für SAD darstellt (Pail et al. 2011), sollte auch Psychopharmakotherapie sowie weitere nicht-pharmakologische und soziale Interventionen je nach der klinischen Gesamtsituation der PatientInnen eingesetzt werden (Pjrek et al. 2004a). Außerdem können SAD PatientInnen von einer Modifikation ihres Lebensstils durch z. B. gesunde Schlafhygiene, Optimierung von Umgebungslicht, morgendliche Spaziergänge, Winterurlaub, oder Sport im Freien signifikant profitieren (Winkler et al. 2006a).

\section{Lichttherapie}

Im Rahmen einer Lichttherapie wird der für die SAD als ursächlich erachtete Mangel an natürlichem Sonnenlicht direkt beeinflusst (Kasper et al. 1988b). 
Durch die Stimulation lichtempfindlicher Melanopsinhaltiger Ganglienzellen der Netzhaut (Berson et al. 2002; Hattar et al. 2002; Roecklein et al. 2013), welche über den retinohypothalamischen Trakt in den vorderen Hypothalamus projizieren, wird im SCN Glutamat freigesetzt (Michel et al. 2002). Der Wirkmechanismus einer Lichttherapie lässt sich weiters über die Synchronisierung phasenverschobener zirkadianer Rhythmen (Lewy et al. 2007), die Unterdrückung einer übermäßigen Melatoninproduktion (Partonen et al. 1997; Pereira et al. 2017), sowie die Normalisierung dysregulierter monoaminerger Neurotransmittersysteme (Neumeister et al. 2001; Spies et al. 2018) und die Beeinflussung veränderter Immunfunktionen (Stastny et al. 2003) erklären. In Zusammenhang mit der Phasenverschiebungshypothese zeigte sich die morgendliche Lichttherapie gegenüber einer späteren Anwendung wiederholt überlegen (Terman et al. 2001; Winkler et al. 2005; Pail et al. 2011).

Im Rahmen einer Lichttherapie, wobei ein sehr helles weißes Licht ohne schädlichen UV-Anteil angewendet wird, zeigen viele PatientInnen eine spürbare Reduktion der depressiven Symptome bereits innerhalb von 4-6 Tagen, während einige erst nach etwa 4 Wochen ansprechen (Pail et al. 2011). Eine statistisch signifikante Überlegenheit der Lichttherapie gegenüber Placebo wurde bei PatientInnen mit einer SAD wiederholt berichtet und rezent durch eine Meta-Analyse der Universitätsklinik für Psychiatrie und Psychotherapie in Wien bestätigt (Pjrek et al. 2020). Während das Vorhandensein atypischer Symptome, wie z. B. Hyperphagie, Hypersomnie und Kohlenhydratheisshunger, sowie auch ein jüngeres Lebensalter mehrfach als Prädiktoren für ein gutes Ansprechen auf die Lichttherapie beschrieben wurden, wies sich die Komorbidität mit einer Persönlichkeitsstörung als ein negativer Prädiktor aus (Terman et al. 1996). Nachdem wiederholte Beobachtungen zeigten, dass es bei SAD PatientInnen nach Absetzen von Lichttherapie rasch zu einem Rückfall kommen kann, sollte Lichttherapie während der dunklen Monate, meistens von Spätsommer bis Ende Februar, einmal täglich für mindestens 30 Minuten angewendet werden (Pail et al. 2011; Nussbaumer et al. 2015).

Anhand aktueller Empfehlungen von internationalen Richtlinien sollten SAD PatientInnen instruiert werden, in einer gemütlichen Sitzposition in Richtung der Lichtlampe, welche eine Lichtstärke von ca. 10000 lux aufweist, mit einem Abstand von etwa $60-80 \mathrm{~cm} \mathrm{zu}$ schauen (Pail et al. 2011). Nachdem das Licht vermutlich direkt über die Augen das Gehirn beeinflusst, sollten die PatientInnen ca. einmal pro Minute kurz direkt in die Lampe blicken, und auf Augenschließen und/oder Tragen von Sonnenbrillen verzichten (Kasper 1994). Obwohl die Lichttherapie als eine sehr gut verträgliche Therapiemethode bezeichnet werden kann, kann es im Rahmen einer täglichen Anwendung hin und wieder zu Kopfschmerzen, Augenüberlastung, Irritabilität oder Schlafstörung kommen (Pail et al. 2011). Sehr selten wurden Menstruationsbeschwerden (Pjrek et al. 2004b), ein Stimmungsswitch in eine Hypomanie sowie erhöhte Suizidalität (Praschak-Rieder et al. 1997) beobachtet. Die Tabelle 1 bietet eine Übersicht wichtiger Informationen zur korrekten Anwendung einer Lichtlampe im Sinne einer effektiven Lichttherapie.

\section{PSYCHOPHARMAKOTHERAPIE}

Neben der Lichttherapie stellen moderne Antidepressiva eine effektive Therapiemethode der SAD dar, welche eingesetzt werden sollten, wenn Lichttherapie aus Zeitmangel nicht durchgeführt werden kann, oder wenn es unter Lichttherapie zu keinem ausreichenden Ansprechen kam (Pjrek et al. 2005). Eine kombinierte Anwendung von Lichttherapie und Psychopharmakotherapie ist sehr gut möglich (Pjrek et al. 2004a).

In Österreich stehen uns derzeit mehrere moderne und sehr gut verträgliche antidepressiv wirkende Substanzen zur Verfügung, welche je nach individueller Symptomatik sowie Nebenwirkungsprofil und Interaktionspotential effektiv eingesetzt werden können. In der Behandlung der SAD haben sich bisher vor allem selektive SerotoninWiederaufnahme-Hemmer (SSRIs), Noradrenalin-Wiederaufnahme Hemmer (NARI), sowie der Noradrenalin-Dopamin-Wiederaufnahme-Hemmer (NDRI), Bupropion, und auch Agomelatin, welches seine antidepressive Effektivität über den Melatoninrezeptor-1/2-Agonismus und gleichzeitig über den Serotonin-2C-Rezeptor-Antagonismus entfaltet, und eine gute Wirksamkeit auf den SchlafWach Rhythmus aufweist, bewährt (Praschak-Rieder \& Willeit 2003; Pjrek et al. 2007; Pjrek et al. 2009). Das ebenfalls über das melatonerge System wirksame retardierte Melatonin hat seine günstige schlaffördernde Wirkung in klinischen Studien nachgewiesen und wird derzeit hauptsächlich für die Behandlung der primären Insomnie angewendet (Quera-Salva \& Claustrat 2018).

\section{Wachtherapie}

Das Konzept der Wachtherapie bzw. des therapeutischen Schlafentzuges bei der depressiven Störung wurde in Zusammenhang allgemein bekannter Beobachtung einer euphorischen Grundstimmung nach Schlafentzug sowie eines für die depressive Störung typischen frühmorgendlichen Erwachens mit Verkürzung des chronobiologischen Tages implementiert (Gerner et al. 1979; Lehofer et al. 2010). Während eines totalen Schlafentzugs werden PatientInnen angeleitet, die ganze Nacht vollkommen auf den Schlaf zu verzichten, während sie beim einem partiellen Schlafentzug bis 1 Uhr in der Früh schlafen und den Rest der Nacht wach bleiben (Benedetti \& Colombo 2011). Bei etwa 60\% der PatientInnen kommt es bereits am nächsten Morgen zu einer deutlichen Reduktion der depressiven Symptomatik. Da jedoch bei vielen PatientInnen nach dem nächsten Schlaf ein Rückfall beobachtet wurde, wird die Fortführung der Wachtherapie im Sinne eines langsamen Einschleichens des Schlafrhythmus zur Schlafphasenvorverlagerung empfohlen, um die Rückfallsneigung zu minimieren (Benedetti \& Colombo 2011). 
Tabelle 1. Wichtige Hinweise für die korrekte Verwendung einer Lichtlampe im Sinne einer effektiven Lichttherapie

\begin{tabular}{l} 
Entscheidende Parameter \\
\hline Art des Lichts \\
Lichtstärke der Lichtlampe \\
Anwendungsdauer
\end{tabular}

Anwendungszeit

Setting während einer Lichttherapie

Abstand zur Lichtlampe

Verhalten während einer Lichttherapie

Wirkungseintritt

Wirkungsdauer

Verträglichkeit

Positive Prädikatoren für ein gutes Therapieansprechen

Negative Prädikatoren für ein gutes Therapieansprechen

Bei Nichtansprechen Empfehlung

Weißes Licht (ohne spezielle Präferenz einer Wellenlänge) ohne schädlichen UV-Anteil

Lampen, die UV-Licht ausstrahlen (z. B. Solarien, Gesichtsbräuner), sind ungeeignet.

10.000 Lux

1x täglich für etwa 30 Minuten

(bei der saisonal abhängigen affektiven Störung 1x täglich für etwa 30

Minuten während der dunklen Monate, meistens von Spätsommer bis Ende Februar)

Morgens (nach dem Aufstehen)

Gemütliche Sitzposition

Beschäftigung im Sitzen (z. B. Lesen, Telefonieren, Essen) möglich $60-80 \mathrm{~cm}$

Etwa 1x pro Minute kurz direkt in die Lichtlampe blicken

Augenschließen bzw. Tragen einer Sonnenbrille verhindert die

Wirksamkeit.

Innerhalb weniger Tage

Nach Beendigung der Lichttherapie lässt die antidepressive Wirksamkeit rasch nach.

Sehr gute Verträglichkeit

Im Rahmen einer täglichen Anwendung zählen Kopfschmerzen, Augenüberlastung, Irritabilität, oder Schlafstörung zu potentiellen Nebenwirkungen.

Selten können Menstruationsbeschwerden, Stimmungsswitch in eine Hypomanie, und sehr selten erhöhte Suizidalität auftreten.

Vorhandensein atypischer Symptome, wie z. B. Hyperphagie, Hypersomnie und Kohlenhydratheisshunger, sowie auch ein jüngeres Lebensalter.

Komorbidität mit Persönlichkeitsstörungen

Kombination mit weiteren effektiven antidepressiv-wirksamen Therapiemethoden wie z. B. Psychopharmakotherapie, Wachtherapie

Die vorliegende Tabelle bietet eine Übersicht wichtiger Hinweise für die korrekte Verwendung einer Lichtlampe im Sinne einer effektiven Lichttherapie, welche anhand von Ergebnissen der derzeit verfügbaren klinischen Studien, systematischen Reviews, Metaanalysen, und im Einklang mit aktuellen international anerkannten Richtlinien erstellt wurde (Kasper 1994; Terman et al. 1996; Praschak-Rieder et al. 1997; Praschak-Rieder et al. 1999; Praschak-Rieder \& Willeit 2003; Pjrek et al. 2004a; Pjrek et al. 2004b; Winkler et al. 2005; Winkler et al. 2006a; Lehofer et al. 2010; Pail et al. 2011; Nussbaumer et al. 2015; Pjrek et al. 2020).

Während klinische Phänomene wie z. B. Tagesschwankungen mit morgendlichem Pessimum, melancholische Züge, und/oder die Diagnose einer bipolaren Depression als Prädiktoren für ein gutes Ansprechen auf Wachtherapie beschrieben wurden, zeigten Alter, Geschlecht, Schweregrad und Dauer der aktuellen depressiven Episode, sowie Anzahl der früheren Episoden, deren Vorbehandlungen und auch die Erwartungshaltung der PatientInnen keinen relevanten Einfluss (Benedetti 2012). Da sich die Wirksamkeit der sowohl totalen als auch partiellen Wachtherapie als vergleichbar zeigte, sollte in der klinischen Routine vorwiegend die partielle Methode angewendet werden (Benedetti \& Colombo 2011). Die Effektivität einer Wachtherapie kann durch eine kombinierte Anwendung mit einer morgendlichen Lichttherapie und antidepressiv wirkender Psychopharmakotherapie erhöht werden (Praschak-Rieder et al. 1999).

\section{CONCLUSIO}

Die unbehandelte SAD wurde schon in den letzten Jahrhunderten als eine chronobiologische Störung verstanden, welche nach Remission zwar nicht mehr vorhanden ist, jedoch ein hohes Rückfallrisiko aufweist. Die positiven Effekte von Licht auf solche jahreszeitlichen Schwankungen von Stimmung, Antrieb, Energieniveau, Schlafdauer, Essverhalten, Körpergewicht sowie von weiteren wichtigen psychischen und körperlichen Funktionen wurden schon in der Antike 
beschrieben. Nachdem in den 1980er-Jahren das Konzept der SAD als eine Störung des zirkadianen Rhythmus vorgestellt wurde, welche durch Mangel an Umgebungslicht in den dunkleren Jahreszeiten hervorgerufen wird, wurde die Lichttherapie bei PatientInnen mit SAD zunehmend untersucht, die sich inzwischen als Monotherapie oder in Kombination mit Psychopharmakotherapie und/oder Wachtherapie in der klinischen Routine erfolgreich etablieren konnte (Rosenthal et al. 1984, Pail et al. 2011, Pjrek et al. 2020).

Durch den Einsatz moderner bildgebender Verfahren konnten Störungen chronobiologischer Rhythmen, welche sich klinisch häufig durch z. B. Appetitsteigerung, Kohlehydratheißhunger, Gewichtszunahme, Gefühl von bleierner Schwere, oder Hypersomnie manifestieren können, mit Veränderungen auf der Neurorezeptor- und Neurotransmitterebene assoziiert werden, welche sich nach erfolgreicher Anwendung der o. g. Behandlungsmöglichkeiten sowie nach Spontanremission wieder normalisieren (Neumeister et al. 2001, Praschak-Rieder \& Willeit 2012). Nachdem eine solche chronobiologische Dysregulation zu den Hauptsymptomen der SAD zählt, mit erhöhten Krankenständen einhergeht (Winkler et al. 2019), und darüber hinaus durch z. B. Schlafstörungen oder Irritabilität oft dem Beginn einer depressiven Episode vorausgeht und somit das Risiko für Suizidalität erhöhen kann (Linnoila \& Virkkunen 1992), muss das Erkennen pathologisch veränderter chronobiologischer Rhythmen und deren adäquate Therapie (NussbaumerStreit et al. 2018) einen festen Bestandteil in der modernen psychiatrischen Behandlung darstellen.

\section{Acknowledgements:}

The authors would like to thank all colleagues from the Department of Psychiatry and Psychotherapy at the Medical University of Vienna involved in the SAD research (Prof. Nicole Praschak-Rieder, Prof. Matthäus Willeit, Prof. Edda Winkler-Pjrek, Prof. Dietmar Winkler, Doz. DDr. Marie Spies, Dr. Michaela Elena Friedrich, Dr. Gerald Pail)."

\section{Conflict of interest:}

DDr. Bartova has received travel grants and consultant/speaker honoraria from AOP Orphan, Medizin Medien Austria, Vertretungsnetz, Schwabe Austria, Janssen and Angelini. Within the last three years, Prof. Kasper has received grants/research support, consulting fees, and/or honoraria from Angelini, Celegne $\mathrm{GmbH}$, Eli Lilly, Janssen-Cilag Pharma $\mathrm{GmbH}$, KRKA-Pharma, Lundbeck A/S, Mundipharma, Neuraxpharm, Pfizer, Sanofi, Schwabe, Servier, Shire, Sumitomo Dainippon Pharma Co. Ltd., sun Pharma and Takeda."

\section{Contribution of individual authors:}

Lucie Bartova \& Siegfried Kasper conceptualized the review.

Lucie Bartova provided the first draft of the manuscript that was critically revised by Prof. Kasper.

Both authors have approved the final manuscript.

\section{References}

1. APA: Diagnostic and statistical manual of mental disorders: DSM-5. American Psychiatric Association, 2013

2. Bach $H$ \& Arango $V$ : Neuroanatomy of Serotonergic Abnormalities in Suicide. In Dwivedi, $Y$. (ed) The Neurobiological Basis of Suicide, Boca Raton (FL), 2012

3. Benedetti F: Antidepressant chronotherapeutics for bipolar depression. Dialogues Clin Neurosci 2012; 14:401-411

4. Benedetti $F \&$ Colombo $C$ : Sleep deprivation in mood disorders. Neuropsychobiology 2011; 64:141-151

5. Berson DM, Dunn FA \& Takao M: Phototransduction by retinal ganglion cells that set the circadian clock. Science 2002; 295:1070-1073

6. Chotai J, Smedh K, Johansson C, Nilsson LG \& Adolfsson R: An epidemiological study on gender differences in selfreported seasonal changes in mood and behaviour in a general population of northern Sweden. Nord J Psychiatry 2004; 58:429-437

7. Friedman E, Gyulai L, Bhargava M, Landen M, Wisniewski S, Foris J, Ostacher M, Medina R \& Thase M: Seasonal changes in clinical status in bipolar disorder: a prospective study in 1000 STEP-BD patients. Acta Psychiatr Scand 2006; 113:510-517

8. Gerner RH, Post RM, Gillin JC \& Bunney WE, Jr: Biological and behavioral effects of one night's sleep deprivation in depressed patients and normals. J Psychiatr Res 1979; 15:21-40

9. Hardin TA, Wehr TA, Brewerton T, Kasper S, Berrettini W, Rabkin $J$ \& Rosenthal NE: Evaluation of seasonality in six clinical populations and two normal populations. J Psychiatr Res $1991 ; 25: 75-87$

10. Harris $S \&$ Dawson-Hughes B: Seasonal mood changes in 250 normal women. Psychiatry Res 1993; 49:77-87

11. Hattar S, Liao HW, Takao M, Berson DM \& Yau KW: Melanopsin-containing retinal ganglion cells: architecture, projections, and intrinsic photosensitivity. Science 2002; 295:1065-1070

12. Hegde AL \& Woodson H: Prevalence of seasonal changes in mood and behavior during the winter months in central Texas. Psychiatry Res 1996; 62:265-271

13. Kasper S: Diagnosis, epidemiology and therapy of seasonal depression. Nervenarzt 1994; 65:69-72

14. Kasper S, Wehr TA, Bartko JJ, Gaist PA \& Rosenthal NE: Epidemiological findings of seasonal changes in mood and behavior. A telephone survey of Montgomery County, Maryland. Archives of general psychiatry 1989; 46:823-833

15. Kasper S, Wehr TA \& Rosenthal NE: Season-related forms of depression. I. Principles and clinical description of the syndrome. Nervenarzt 1988a; 59:191-199

16. Kasper S, Wehr TA \& Rosenthal NE: Season-related forms of depression. II. Modification by phototherapy and biological results. Nervenarzt 1988b; 59:200-214

17. Kudlow PA, Cha DS, Lam RW \& McIntyre RS: Sleep architecture variation: a mediator of metabolic disturbance in individuals with major depressive disorder. Sleep Med 2013; 14:943-949

18. Lambert, G., Reid, C., Kaye, D., Jennings, G. \& Esler, M. (2003) Increased suicide rate in the middle-aged and its association with hours of sunlight. Am J Psychiatry, 160, 793795

19. Lambert, G.W., Reid, C., Kaye, D.M., Jennings, G.L. \& Esler M.D. (2002) Effect of sunlight and season on serotonin turnover in the brain. Lancet, 360, 1840-1842.

20. Lehofer M, Pail G, Kasper S, Czermak C, Erfurth A, Högl B, Huf W, Praschak-Rieder N, Saletu M \& Willeit M: Chronopsychiatrie. Vienna, Austria, 2010 
21. Levitt, A.J. \& Boyle, M.H. (2002) The impact of latitude on the prevalence of seasonal depression. Can J Psychiatry, 47, 361-367

22. Levitt, A.J., Boyle, M.H., Joffe, R.T. \& Baumal, Z. (2000) Estimated prevalence of the seasonal subtype of major depression in a Canadian community sample. Can $J$ Psychiatry, 45, 650-654.

23. Lewy, A.J., Rough, J.N., Songer, J.B., Mishra, N., Yuhas, K. \& Emens, J.S. (2007) The phase shift hypothesis for the circadian component of winter depression. Dialogues Clin Neurosci, 9, 291-300.

24. Liebmann PM, Wolfler A, Felsner P, Hofer D \& Schauenstein K: Melatonin and the immune system. Int Arch Allergy Immunol 1997; 112:203-211

25. Linkowski P, Mendlewicz J, Kerkhofs M, Leclercq R, Golstein $J$, Brasseur M, Copinschi G \& Van Cauter E: 24-hour profiles of adrenocorticotropin, cortisol, and growth hormone in major depressive illness: effect of antidepressant treatment. J Clin Endocrinol Metab 1987; 65:141-152

26. Linnoila, V.M. \& Virkkunen, M. (1992) Aggression, suicidality, and serotonin. J Clin Psychiatry, 53 Suppl, 46-51.

27. Mendoza, J. (2019) Circadian insights into the biology of depression: Symptoms, treatments and animal models. Behav Brain Res, 376, 112186

28. Michel, S., Itri, J. \& Colwell, C.S. (2002) Excitatory mechanisms in the suprachiasmatic nucleus: the role of AMPA/KA glutamate receptors. J Neurophysiol, 88, 817-828.

29. Murray G: Diurnal mood variation in depression: a signal of disturbed circadian function? J Affect Disord 2007; 102:47-53

30. Neumeister, A., Konstantinidis, A., Praschak-Rieder, N., Willeit, M., Hilger, E., Stastny, J. \& Kasper, S. (2001) Monoaminergic function in the pathogenesis of seasonal affective disorder. Int J Neuropsychopharmacol, 4, 409-420.

31. Nussbaumer, B., Kaminski-Hartenthaler, A., Forneris, C.A., Morgan, L.C., Sonis, J.H., Gaynes, B.N., Greenblatt, A., Wipplinger, J., Lux, L.J., Winkler, D., Van Noord, M.G., Hofmann, J. \& Gartlehner, G. (2015) Light therapy for preventing seasonal affective disorder. Cochrane Database Syst Rev, CD011269.

32. Nussbaumer-Streit, B., Pjrek, E., Kien, C., Gartlehner, G., Bartova, L., Friedrich, M.E., Kasper, S. \& Winkler, D. (2018) Implementing prevention of seasonal affective disorder from patients' and physicians' perspectives - a qualitative study. BMC Psychiatry, 18, 372

33. Okawa, M., Shirakawa, S., Uchiyama, M., Oguri, M., Kohsaka, M., Mishima, K., Sakamoto, K., Inoue, H., Kamei, K. \& Takahashi, K. (1996) Seasonal variation of mood and behaviour in a healthy middle-aged population in Japan. Acta Psychiatr Scand, 94, 211-216.

34. Pail G, Huf W, Pjrek E, Winkler D, Willeit M, PraschakRieder $N$ \& Kasper S: Bright-light therapy in the treatment of mood disorders. Neuropsychobiology 2011; 64:152-162

35. Partonen, T., Vakkuri, O. \& Lonnqvist, J. (1997) Suppression of melatonin secretion by bright light in seasonal affective disorder. Biol Psychiatry, 42, 509-513.

36. Peeters, F., Berkhof, J., Delespaul, P., Rottenberg, J. \& Nicolson, N.A. (2006) Diurnal mood variation in major depressive disorder. Emotion, 6, 383-391

37. Pereira JC Jr, Pradella Hallinan $M \&$ Alves RC: Secondary to excessive melatonin synthesis, the consumption of tryptophan from outside the blood-brain barrier and melatonin over-signaling in the pars tuberalis may be central to the pathophysiology of winter depression. Med Hypotheses 2017; 98:69-75

38. Perry, J.A., Silvera, D.H., Rosenvinge, J.H., Neilands, T. \& Holte, A. (2001) Seasonal eating patterns in Norway: a nonclinical population study. Scand J Psychol, 42, 307-312
39. Pjrek, E., Friedrich, M.E., Cambioli, L., Dold, M., Jager, F., Komorowski, A., Lanzenberger, R., Kasper, S. \& Winkler, D. (2020) The Efficacy of Light Therapy in the Treatment of Seasonal Affective Disorder: A Meta-Analysis of Randomized Controlled Trials. Psychother Psychosom, 89, 17-24

40. Pjrek E, Konstantinidis A, Assem-Hilger E, Praschak-Rieder $N$, Willeit M, Kasper $S$ \& Winkler D: Therapeutic effects of escitalopram and reboxetine in seasonal affective disorder: a pooled analysis. J Psychiatr Res 2009; 43:792-797

41. Pjrek E., Winkler, D. \& Kasper, S. (2005) Pharmacotherapy of seasonal affective disorder. CNS Spectr, 10, 664-669; quiz 672

42. Pjrek E, Winkler D, Konstantinidis A, Willeit M, PraschakRieder $N$ \& Kasper S: Agomelatine in the treatment of seasonal affective disorder. Psychopharmacology (Berl) 2007; 190:575-579

43. Pjrek, E., Winkler, D., Stastny, J., Konstantinidis, A., Heiden, A. \& Kasper, S. (2004a) Bright light therapy in seasonal affective disorder--does it suffice? Eur Neuropsychopharmacol, 14, 347-351.

44. Pjrek, E., Winkler, D., Willeit, M., Konstantinidis, A., Thierry, N. \& Kasper, S. (2004b) Menstrual disturbances a rare sideeffect of bright-light therapy. Int J Neuropsychopharmacol, 7 , 239-240.

45. Praschak-Rieder, N., Neumeister, A., Hesselmann, B., Willeit, M., Barnas, C. \& Kasper, S. (1997) Suicidal tendencies as a complication of light therapy for seasonal affective disorder: a report of three cases. J Clin Psychiatry, 58, 389-392

46. Praschak-Rieder, N. \& Willeit, M. (2003) Treatment of seasonal affective disorders. Dialogues Clin Neurosci, 5, 389398

47. Praschak-Rieder, N. \& Willeit, M. (2012) Imaging of seasonal affective disorder and seasonality effects on serotonin and dopamine function in the human brain. Curr Top Behav Neurosci, 11, 149-167

48. Praschak-Rieder N, Willeit M, Neumeister A, Hilger E \& Kasper S: Therapeutic sleep deprivation and phototherapy. Wien Med Wochenschr 1999; 149:520-524

49. Praschak-Rieder, N., Willeit, M., Wilson, A.A., Houle, S. \& Meyer, J.H. (2008) Seasonal variation in human brain serotonin transporter binding. Archives of general psychiatry, 65, 1072-1078

50. Praschak-Rieder N, Willeit M, Winkler D, Neumeister A, Hilger E, Zill P, Hornik K, Stastny J, Thierry N, Ackenheil M, Bondy $B$ \& Kasper S: Role of family history and 5-HTTLPR polymorphism in female seasonal affective disorder patients with and without premenstrual dysphoric disorder. Eur Neuropsychopharmacol 2002; 12:129-134

51. Praschak-Rieder N, Willeit M, Zill P, Winkler D, Thierry N, Konstantinidis A, Masellis M, Basile VS, Bondy B, Ackenheil $M$, Neumeister A, Kaplan AS, Kennedy JL, Kasper $S \&$ Levitan R: A Cys 23-Ser 23 substitution in the 5-HT(2C) receptor gene influences body weight regulation in females with seasonal affective disorder: an Austrian-Canadian collaborative study. J Psychiatr Res 2005a; 39:561-567

52. Praschak-Rieder N, Wilson AA, Hussey D, Carella A, Wei C, Ginovart N, Schwarz MJ, Zach J, Houle S \& Meyer JH: Effects of tryptophan depletion on the serotonin transporter in healthy humans. Biol Psychiatry 2005b; 58:825-830

53. Quera-Salva MA \& Claustrat B: Melatonin: Physiological and pharmacological aspects related to sleep: The interest of a prolonged-release formulation (Circadin $((R)))$ in insomnia. Encephale 2018; 44:548-557

54. Roecklein, K.A., Wong, P.M., Miller, M.A., Donofry, S.D., Kamarck, M.L. \& Brainard, G.C. (2013) Melanopsin, photosensitive ganglion cells, and seasonal affective disorder. Neurosci Biobehav Rev, 37, 229-239 
55. Rohan, K.J. \& Sigmon, S.T. (2000) Seasonal mood patterns in a northeastern college sample. J Affect Disord, 59, 85-96.

56. Rosenthal, N.E., Sack, D.A., Gillin, J.C., Lewy, A.J., Goodwin, F.K., Davenport, Y., Mueller, P.S., Newsome, D.A. \& Wehr, T.A. (1984) Seasonal affective disorder. A description of the syndrome and preliminary findings with light therapy. Archives of general psychiatry, 41, 72-80.

57. Sebestyen, B., Rihmer, Z., Balint, L., Szokontor, N., Gonda, X., Gyarmati, B., Bodecs, T. \& Sandor, J. (2010) Gender differences in antidepressant use-related seasonality change in suicide mortality in Hungary, 1998-2006. World J Biol Psychiatry, 11, 579-585

58. Souetre, E., Salvati, E., Belugou, J.L., Pringuey, D., Candito, M., Krebs, B., Ardisson, J.L. \& Darcourt, G. (1989) Circadian rhythms in depression and recovery: evidence for blunted amplitude as the main chronobiological abnormality. Psychiatry Res, 28, 263-278

59. Spies M, James GM, Vraka C, Philippe C, Hienert M, Gryglewski G, Komorowski A, Kautzky A, Silberbauer L, Pichler V, Kranz GS, Nics L, Balber T, Baldinger-Melich P, Vanicek T, Spurny B, Winkler-Pjrek E, Wadsak W, Mitterhauser $M$, Hacker $M$, Kasper S, Lanzenberger $R$ \& Winkler D: Brain monoamine oxidase A in seasonal affective disorder and treatment with bright light therapy. Transl Psychiatry 2018; 8:198

60. Stastny, J., Konstantinidis, A., Schwarz, M.J., Rosenthal, N.E., Vitouch, O., Kasper, S. \& Neumeister, A. (2003) Effects of tryptophan depletion and catecholamine depletion on immune parameters in patients with seasonal affective disorder in remission with light therapy. Biol Psychiatry, 53, 332-337

61. Terman, J.S., Terman, M., Lo, E.S. \& Cooper, T.B. (2001) Circadian time of morning light administration and therapeutic response in winter depression. Archives of general psychiatry, 58, 69-75

62. Terman, M., Amira, L., Terman, J.S. \& Ross, D.C. (1996) Predictors of response and nonresponse to light treatment for winter depression. Am J Psychiatry, 153, 1423-1429

63. Vyssoki, B., Kapusta, N.D., Praschak-Rieder, N., Dorffner, G. \& Willeit, M. (2014) Direct effect of sunshine on suicide. JAMA psychiatry, 71, 1231-1237

64. Vyssoki B, Praschak-Rieder N, Sonneck G, Bluml V, Willeit M, Kasper $S$ \& Kapusta ND: Effects of sunshine on suicide rates. Comprehensive psychiatry 2012; 53:535-539

65. Wehr TA, Duncan WC, Jr, Sher L, Aeschbach D, Schwartz PJ, Turner EH, Postolache TT \& Rosenthal NE: A circadian signal of change of season in patients with seasonal affective disorder. Archives of general psychiatry 2001; 58:1108-1114
66. Wehr TA, Sack DA \& Rosenthal NE: Seasonal affective disorder with summer depression and winter hypomania. Am J Psychiatry 1987; 144:1602-1603

67. Willeit, M., Praschak-Rieder, N., Neumeister, A., Pirker, W., Asenbaum, S., Vitouch, O., Tauscher, J., Hilger, E., Stastny, J., Brucke, T. \& Kasper, S. (2000) [123I]-beta-CIT SPECT imaging shows reduced brain serotonin transporter availability in drug-free depressed patients with seasonal affective disorder. Biol Psychiatry, 47, 482-489

68. Willeit M, Praschak-Rieder N, Zill P, Neumeister A, Ackenheil $M$, Kasper $S$ \& Bondy B: C825T polymorphism in the $G$ protein beta3-subunit gene is associated with seasonal affective disorder. Biol Psychiatry 2003; 54:682-686

69. Willeit M, Sitte HH, Thierry N, Michalek K, Praschak-Rieder $N$, Zill P, Winkler D, Brannath $W$, Fischer MB, Bondy B, Kasper $S$ \& Singer EA: Enhanced Serotonin Transporter Function during Depression in Seasonal Affective Disorder. Neuropsychopharmacology 2008; 33; 1503-1513

70. Winkler, D., Pjrek, E., Iwaki, R. \& Kasper, S. (2006a) Treatment of seasonal affective disorder. Expert Rev Neurother, 6, 1039-1048

71. Winkler D, Pjrek E, Konstantinidis A, Praschak-Rieder N, Willeit M, Stastny J \& Kasper S: Anger attacks in seasonal affective disorder. Int J Neuropsychopharmacol 2006b; 9:215-219

72. Winkler D, Pjrek E, Praschak-Rieder N, Willeit M, Pezawas L, Konstantinidis A, Stastny $J$ \& Kasper S: Actigraphy in patients with seasonal affective disorder and healthy control subjects treated with light therapy. Biol Psychiatry 2005; 58:331-336

73. Winkler D, Praschak-Rieder N, Willeit M, Lucht MJ, Hilger E, Konstantinidis A, Stastny J, Thierry N, Pjrek E, Neumeister A, Moller HJ \& Kasper S: Seasonal affective depression in 2 German speaking university centers: Bonn, Vienna. Clinical and demographic characteristics. Nervenarzt 2002a; 73:637-643

74. Winkler D, Reichardt B, Kranz GS, Bartova L, Kasper S \& Pjrek E: Seasonality of antidepressant prescriptions and sick leaves. J Psychiatr Res 2019; 111:128-133

75. Winkler D, Willeit M, Praschak-Rieder N, Lucht MJ, Hilger E, Konstantinidis A, Stastny J, Thierry N, Pjrek E, Neumeister $A$, Moller HJ \& Kasper S: Changes of clinical pattern in seasonal affective disorder (SAD) over time in a Germanspeaking sample. Eur Arch Psychiatry Clin Neurosci 2002b; 252:54-62

76. Wittmann $M$, Schreiber $W$, Landgrebe $M$ \& Hajak G: Circadian rhythms and depression. Fortschr Neurol Psychiatr 2018; $86: 308-318$
Correspondence:

Prof. Siegfried Kasper, MD, Professor Emeritus

Center for Brain Research, Medical University of Vienna

Spitalgasse 4, A-1090 Vienna, Austria

E-mail: siegfried.kasper@meduniwien.ac.at 